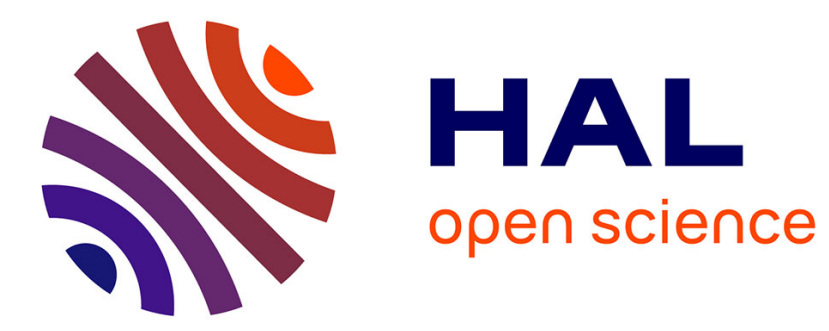

\title{
Robust steering control of hot strip mill
}

\author{
Ivan Malloci, Jamal Daafouz, Claude Iung, Rémi Bonidal, Patrick Szczepanski
}

\section{To cite this version:}

Ivan Malloci, Jamal Daafouz, Claude Iung, Rémi Bonidal, Patrick Szczepanski. Robust steering control of hot strip mill. 10th European Control Conference, ECC'09, Aug 2009, Budapest, Hungary. pp.CDROM. hal-00417791

\section{HAL Id: hal-00417791 \\ https://hal.science/hal-00417791}

Submitted on 16 Sep 2009

HAL is a multi-disciplinary open access archive for the deposit and dissemination of scientific research documents, whether they are published or not. The documents may come from teaching and research institutions in France or abroad, or from public or private research centers.
L'archive ouverte pluridisciplinaire HAL, est destinée au dépôt et à la diffusion de documents scientifiques de niveau recherche, publiés ou non, émanant des établissements d'enseignement et de recherche français ou étrangers, des laboratoires publics ou privés. 


\section{Robust Steering Control of Hot Strip Mill}

Ivan Malloci, Jamal Daafouz, Member, IEEE, Claude Iung, Member, IEEE, Rémi Bonidal and Patrick Szczepanski

\begin{abstract}
In this article, a robust hot strip mill steering control is proposed in order to guarantee the system stability and to improve its performances. The rolling process consists in crushing a metal strip between some rolls for obtaining a product with constant and desired thickness. The quality of the rolled products and the safety of the production process are linked to the lateral movement of the strip in the line. Then, the main task of the steering control consists in reducing this displacement. A hot strip mill treats products with very different characteristics. Then, the products are divided into several families, with reference to their physical characteristics, and the stabilisation problem is formulated as a convex problem. Finally, for each family, a different robust controller is designed. Simulated and experimental results concerning the steering control in the ArcelorMittal hot strip mill of Eisenhüttenstadt are presented.
\end{abstract}

Index Terms - Hot strip mill, Steering control, Singular perturbation, Polytopic uncertainties, $H_{2}$ robust control design, Linear matrix inequalities.

\section{INTRODUCTION}

A hot strip mill is composed by several stands in a line, where each stand is constituted by a set of rolls. The rolling process consists in crushing a metal strip between the rolls for obtaining a product with constant and desired thickness. Furthermore, specific physical characteristics are required for the rolled products. In order to prevent a decrease of the product quality or rolling accidents, the strip has to be maintained on the centre of the mill line. The lateral movement of the strip with reference to the mill axis is called strip off-centre.

The most important contributions on the steering control theory are based on differential force measurements [5], [10], [11] and [12]. The differential force is assumed to be the strip off-centre image. Hence, the stand tilting correction is fixed proportional to the strip off-centre and a PID controller can be used. Nevertheless, the law linking the differential force and the strip off-centre is strongly non-linear. These constraints are taken into account in [3], where a linear quadratic controller is designed for a nominal framework. Since a mill treats products with very different characteristics, this strategy cannot guarantee the same performances for the whole set of rolled products.

This work has been supported by grants from "la région Lorraine, France" and ArcelorMittal Maizières Research.

I. Malloci, J. Daafouz and C. Iung are with the Centre de Recherche en Automatique de Nancy, UMR 7039 CNRS - Nancy Université, ENSEM, 2, Avenue de la forêt de Haye 54516 Vandoeuvre-lès-Nancy, France. Corresponding author: Jamal.Daafouz@ensem.inpl-nancy.fr.

R. Bonidal and P. Szczepanski are with ArcelorMittal Maizières, R\&D Industrial Operations, BP 30320, F-57283 Maizières-lès-Metz Cedex, France.
In this article, we propose a robust controller able to stabilises the mill system, reducing the strip off-centre for the whole set of treated products. The variation of the product parameters is taken into account in the control design: strips are divided into several families, with reference to their thickness and width. For each family, a different controller is designed. A polytopic approach is used to describe the system as a convex problem and then to design a robust controller using LMI technics [2].

The article is organised as follows. In section II, the physical system equations are given. Moreover, the linearised system and the singular perturbation approximation used to design the controller are presented [6]. In section III, the polytopic modeling is introduced. A method to simplify the system description exploiting physical relations between parameters is proposed. In section IV, the robust controller is designed. In section V, simulated and experimental results concerning the ArcelorMittal hot strip mill of Eisenhüttenstadt (Germany) are presented.

\section{SYSTEM DESCRIPTION}

A hot strip mill (HSM) is constituted by $n$ stands (Fig. 1). Each stand contains a set of rolls (composed by two work rolls and two support rolls). Only the case $n=5$ is considered. The generalisation to $n$ stands can be made without any theoretical difficulty.

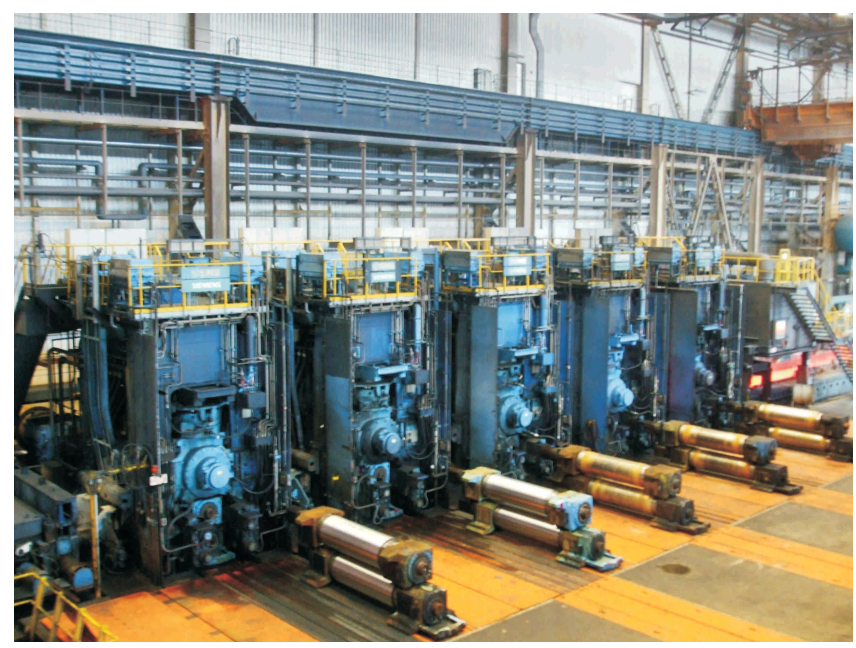

Fig. 1. ArcelorMittal Eisenhüttenstadt HSM view

The superposition of the relations governing the system behaviour yields to the non-linear strip off-centre physical model. 
For each stand $j \in \Upsilon=\{1, \ldots, n\}$, the main physical parameters are the strip width $w_{j}$, the strip thickness $h_{j}$, the back strip tension $T_{j}^{a m}$, the front strip tension $T_{j}^{a v}$, the screw interaxis length $l_{j}^{v}$, the interstand length $l_{j}^{0}$, the work rolls speed $s_{j}$ and the Young's module $E_{j}$. The constants $c_{j}^{f h}, c_{j}^{f T_{a m}}, c_{j}^{f T_{a v}}, c_{j}^{g h}, c_{j}^{g T_{a m}}, c_{j}^{g T_{a v}}, K_{j}^{h}, K_{j}^{f}, K_{j}^{l}, P_{j}$ and $g_{j}$ represent the gradient of the strip parameters. They can be evaluated using numerical algorithms developed by the ArcelorMittal engineers. Furthermore, the main asymmetries are the strip off-centre $Z_{j}$, the strip thickness profile (wedge) $\Delta h_{j}$, the stand tilting $\Delta S_{j}$ and the differential stand stretch $\Delta K_{j}$.

The main equations governing the system are:

- The differential rolling force equation

$$
\begin{aligned}
\Delta P_{j}= & c_{j-1}^{f h} \Delta h_{j-1}+c_{j}^{f h} \Delta h_{j} \\
& +c_{j}^{f T_{a m}} \Delta T_{j}^{a m}+c_{j}^{f T_{a v}} \Delta T_{j}^{a v}
\end{aligned}
$$

- The exit stand wedge equation

$$
\begin{aligned}
\Delta h_{j}= & \left(\frac{w_{j}}{\left(l^{v}\right)_{j}^{2} K_{j}^{h}}+\frac{6 w_{j}}{b_{j}^{2} K_{j}^{f}}\right)\left(\Delta P_{j}+2 P_{j}\right) Z_{j} \\
& +\frac{\Delta P_{j}}{K_{j}^{l}}+\frac{w_{j}}{l_{j}^{v}} \Delta S_{j}-\frac{w_{j}}{l_{j}^{v}\left(K_{j}^{h}\right)^{2}} P_{j} \Delta K_{j}
\end{aligned}
$$

- The angle $\alpha_{j}$ between the strip and the mill axis equation

$$
\begin{aligned}
\dot{\alpha}_{j}= & \frac{s_{j}}{w_{j}}\left(\frac{c_{j}^{g h}}{1+g_{j}}+\frac{1}{h_{j}}\right) \Delta h_{j}+\frac{s_{j}}{w_{j}}\left(\frac{c_{j-1}^{g h}}{1+g_{j}}-\frac{1}{h_{j-1}}\right) \Delta h_{j-1} \\
& +\frac{s_{j} c_{j}^{g T_{a v}}}{w_{j}\left(1+g_{j}\right)} \Delta T_{j}^{a v}+\frac{s_{j} c_{j}^{g T_{a m}}}{w_{j}\left(1+g_{j}\right)} \Delta T_{j}^{a m}
\end{aligned}
$$

- The strip off-centre equation

$$
\dot{Z}_{j}=s_{j} \alpha_{j}
$$

- The front-back differential of strip tension equation

$$
\begin{aligned}
\Delta T_{j}^{a m}= & 3\left(\frac{w_{j} E_{j}}{\left(l_{j}^{0}\right)^{2}}+\frac{T_{j}^{a m}}{w_{j}}\right)\left(Z_{j}-Z_{j-1}\right) \\
& +\frac{w_{j} E_{j}}{l_{j}^{0}}\left(2 \alpha_{j}-\alpha_{j-1}\right)+3 \frac{l_{j}^{0} T_{j}^{a m}}{w_{j}} \alpha_{j} ;
\end{aligned}
$$

- The coupling between two successive stands equation

$$
\Delta T_{j-1}^{a v}=-\Delta T_{j}^{a m}
$$

Two state variables can be associated to each stand: the strip off-centre $Z_{j}$ and the angle $\alpha_{j}$. According to the previous physical equations, the complete process is described by the non-linear continuous-time differential system

$$
\dot{x}=\varphi(x, u, d)
$$

where

$$
x=\left[\alpha_{1}, \ldots, \alpha_{n}, Z_{1}, \ldots, Z_{n}\right]^{\prime} \in \mathbb{R}^{2 n}
$$

is the state, $u \in \mathbb{R}^{r}$ is the control signal (the stand tilting $\Delta S$ ) and $d \in \mathbb{R}^{q}$ the external perturbation, which consists in the strip off-centre in the input of the first stand due to the vibrations of the coilbox (the device used to coil the strips into the finishing train). The other perturbations on the system are negligible with reference to the input strip offcentre. There exist $n$ cameras to measure the state variables $Z_{1}, \ldots, Z_{n}$.

The main task of the steering control consists in reducing the lateral movement of the strip, which can be maintained close to the mill axis modifying the differential rolling force. Since the stand tiltings are bounded, only small deviations around the ideal operating point $\left(\alpha_{j}=Z_{j}=\Delta h_{j}=0\right)$ must be considered. Then, we obtain the following linearised model:

$$
\left\{\begin{array}{l}
\dot{x}=M x+N^{u} u+N^{d} d \\
y=C^{y} x
\end{array}\right.
$$

The HSM system has a two-time scale dynamics: the angles are fast variables with reference to the strip off-centre variables. In this case, some numerical problems can occur due to the stiffness of this kind of structure. Moreover, the system actuators have a limited rate. Then, the dynamics corresponding to the angles cannot be directly controlled.

In this case, the singular perturbation approximation can be used in order to design the controller. It consists in describing the system behaviour in the slow manifold. The differential equations concerning the fast variables are replaced by algebraic equations. Decomposing the system state into the fast and slow components $x=\left[x_{1}^{\prime} x_{2}^{\prime}\right]^{\prime}$, we obtain:

$$
\left\{\begin{array}{l}
\dot{x}_{1}=M_{11} x_{1}+M_{12} x_{2}+N_{1}^{u} u+N_{1}^{d} d \\
\dot{x}_{2}=M_{21} x_{1}+M_{22} x_{2}+N_{2}^{u} u+N_{2}^{d} d \\
y=C_{1}^{y} x_{1}+C_{2}^{y} x_{2} .
\end{array}\right.
$$

Fixing $\dot{x}_{1}=0$,

$$
M_{11} x_{1}+M_{12} x_{2}+N_{1}^{u} u+N_{1}^{d} d=0
$$

gives the slow manifold equation and

$$
\left\{\begin{array}{l}
\dot{x}_{s}=M_{s} x_{s}+N_{s}^{u} u+N_{s}^{d} d \\
y=C_{s}^{y} x_{s}
\end{array}\right.
$$

gives the zero order approximation of the system, where

$$
x_{s}=\left[\begin{array}{lll}
Z_{1} & \ldots & Z_{n}
\end{array}\right]^{\prime} \in \mathbb{R}^{n}
$$

and

$$
\begin{aligned}
& M_{s}=M_{22}-M_{21} M_{11}^{-1} M_{12}, \\
& N_{s}^{u}=N_{2}^{u}-M_{21} M_{11}^{-1} N_{1}^{u}, \\
& N_{s}^{d}=N_{2}^{d}-M_{21} M_{11}^{-1} N_{1}^{d}, \\
& C_{s}=C_{2}-M_{21} M_{11}^{-1} C_{1} .
\end{aligned}
$$

The pair $\left(M_{s}, N_{s}^{u}\right)$ is assumed to be controllable and the state vector $x_{s}$ always available. Moreover, the matrix $M_{11}$ is assumed to be invertible, Hurwitz and it verifies the condition

$$
\max _{\iota} \operatorname{Re}\left(\xi_{\iota}\left\{M_{11}\right\}\right)<\min _{\zeta} \operatorname{Re}\left(\xi_{\zeta}\left\{M_{s}\right\}\right),
$$

with $\iota, \zeta \in \Upsilon$. In this case, the singularly perturbed system (11) gives a good approximation of the original system (9). 


\section{POLYTOPIC MODELING}

The products rolled in a mill have very different physical characteristics. Hence, we have to consider a different uncertainty for each parameter of the product. The uncertain parameters depend on the physical parameters of the rolled strip (width, thickness, temperature, etc.) and on the HSM parameters (roll radius, roll speed, roll force, etc.). The HSM parameters are related to the strip but fixed by an operator. Then, each product is characterised by its strip and HSM parameters. Since products can be very different, several controllers have to be designed in order to obtain the same performances for any product. The whole set of products that can be treated in the mill will be divided into several families, with reference to their physical parameters. For each family, a specific controller will be designed.

In this section, a method to formulate the robust steering control as a convex problem is presented. Furthermore, it is shown how the complexity of the problem can be reduced exploiting the physical relations between the different product parameters. According to the practical implementation, the controller must be designed in the discrete-time. Consider

$$
x(k+1)=\mathcal{A} x(k),
$$

where $\mathcal{A}$ is the polytopic domain

$$
\mathcal{A}=\sum_{\nu=1}^{N_{v}} \lambda_{\nu} A_{\nu},
$$

$\lambda_{\nu}$ denotes the uncertainty and belongs to the unit simplex

$$
\Lambda=\left\{\sum_{\nu=1}^{N_{v}} \lambda_{\nu}=1, \lambda_{\nu} \geq 0\right\}
$$

and $N_{v}$ is the vertices number of the convex hull. The whole set of products is divided into $\mathcal{F}$ families. For each family $f \in \Psi=\{1, \ldots, \mathcal{F}\}$, the vertices number of its convex hull $N_{v}^{f}$, with $\nu \in \Gamma^{f}=\left\{1, \ldots, N_{v}^{f}\right\}$ and the products number $N_{p}^{f}$, with $p \in \Omega^{f}=\left\{1, \ldots, N_{p}^{f}\right\}$, can be defined.

The space dimension $\mathcal{D}$ of the convex hull coincides with the number of uncertain parameters $\mathcal{U}^{p}$. In the considered $\mathrm{HSM}, \mathcal{U}^{p}=70$. Since the state matrices are ill-conditioned, the LMI solvers cannot find any solution, with a problem of this dimension. Then, a simplified system description has to be found. We know that a set of main parameters $\mathcal{U}^{m}$ can be determined. When two products have the same main parameters, the systems have similar dynamics. Let define $\mathcal{U}^{m}=\left\{\mathcal{U}^{p} \backslash \mathcal{U}^{s}\right\}$, where $\mathcal{U}^{s}$ is the set of the remaining parameters (secondary parameters). $\mathcal{U}^{s}$ can be divided into two subsets. The first one can be computed by analytic functions. For example the relation between the roll speed and the output thickness of two adjacent stands is given by the law of matter conservation. The second subset of $\mathcal{U}^{s}$ depends on the operator choice. Nevertheless, for similar strips the operator must use analogous HSM parameters. Consequently, products with similar $\mathcal{U}^{m}$ have also similar $\mathcal{U}^{s}$.

We simulated the system behaviour with different products and we found $\mathcal{U}^{m}=\left\{w, h_{n}, \sigma_{1}^{0}, \sigma_{n}^{0}\right\}$, where $w$ is the strip width, $h_{n}$ is the output thickness of the strip in the last stand and $\sigma_{1}^{0}$ and $\sigma_{n}^{0}$ are the hardness of the strip in the first and in the last stand, respectively. Hence, the system dynamics can be described by a convex hull with dimension $\mathcal{D}=4$.

In order to compute the convex hull, two different strategies have been evaluated. The first one consists in considering each product as a point in the main parameters space. Thus, the corresponding convex hull can be computed, for example, using the free software Qhull [1]. We find at least $N_{v}^{f}=100$.

The second strategy consists in considering the $2^{\mathcal{D}}$ possible combinations of the maximum and minimum values of each dimension. Then, a hyper trapezoid with $N_{v}^{f}=16$ vertices is found. With this approach, the number of vertices can be considerably reduced. This decreases numerical problems which may be very restrictive for LMI solvers. Moreover, products which are not in the database but which could be rolled in the future are taken into account.

For these reasons, we decided to use the second method. In this case, the products corresponding to the convex hull vertices could not be present in the database because the combinations between the main parameters are infinite. Then, in most of the cases, secondary parameters of the products corresponding to the vertices have to be computed. For each family $f$, the set $\mathcal{U}^{s}(\nu, f)$ of the product corresponding to the vertex $\nu$ in the family $f$ can be imposed to be equal to the set $\mathcal{U}^{s}(p, f)$ of the closest product $p$, which is computed by the equation

$$
D_{\min }(\nu, p, f)=\min _{(\nu, p) \in \Gamma_{\nu}^{f} \times \Omega^{f}}\{D(\nu, p, f)\}
$$

with

$$
\begin{aligned}
D(\nu, p, f)= & {\left[(w(\nu, f)-w(p, f))^{2}+\left(h_{s}(\nu, f)-h_{s}(p, f)\right)^{2}+\right.} \\
& \left.\left(\sigma_{1}^{0}(\nu, f)-\sigma_{1}^{0}(p, f)\right)^{2}+\left(\sigma_{n}^{0}(\nu, f)-\sigma_{n}^{0}(p, f)\right)^{2}\right]^{\frac{1}{2}}
\end{aligned}
$$

$\forall(\nu, p, f) \in \Gamma_{\nu}^{f} \times \Omega^{f} \times \Psi . D(\nu, p, f)$ represents the distance, with reference to the set $\mathcal{U}^{m}$, between the vertex $\nu$ and the product $p$ in the family $f$. To compute it, all the parameters are normalised beforehand into the interval $[-1,1]$. The approximately trapezoidal shape of the database guarantees that each vertex is close at least to a product. Then, the proposed solution assures a good approximation.

\section{IV. $H_{2}$ CONTROL DESIGN}

In this section, we describe the robust controller designed for each family $f \in \Psi$. For simplicity, the index $f$ is omitted. Consider the uncertain discrete-time linear system

$$
\left\{\begin{array}{l}
x(k+1)=\mathcal{A} x(k)+\mathcal{B}^{u} u(k)+\mathcal{B}^{d} d(k) \\
z(k)=C^{z} x(k)+D^{z u} u(k) \\
y(k)=C^{y} x(k)
\end{array}\right.
$$

where $x \in \mathbb{R}^{n}$ is the state, $u \in \mathbb{R}^{r}$ is the control signal, $d \in$ $\mathbb{R}^{q}$ is the external perturbation, $y \in \mathbb{R}^{m}$ is the measured output and $z \in \mathbb{R}^{m}$ is the controlled output. Let define the convex hulls

$$
\mathcal{A}=\sum_{\nu=1}^{N_{v}} \lambda_{\nu}^{A} A_{\nu}
$$




$$
\mathcal{B}^{u}=\sum_{v=1}^{N_{v}} \lambda_{\nu}^{B^{u}} B_{\nu}^{u}
$$

and

$$
\mathcal{B}^{d}=\sum_{\nu=1}^{N_{v}} \lambda_{\nu}^{B^{d}} B_{\nu}^{d},
$$

with $\left\{\lambda_{\nu}^{A}, \lambda_{\nu}^{B^{u}}, \lambda_{\nu}^{B^{d}}\right\} \in \Lambda$. A $H_{2}$ LMI-based controller is designed. It provides a solution taking a priori into account the uncertainties and minimising the effects of the external perturbations. Given the control law

$$
u(k)=K x(k)
$$

and the closed loop matrices $A_{\nu}^{c l}=A_{\nu}+B_{\nu}^{u} K$ and $C^{c l}=$ $C^{z}+D^{z u} K$, the transfer matrix between $z$ and $d$ is

$$
T_{\nu}^{d z}(\varsigma)=C^{c l}\left(\varsigma I-A_{\nu}^{c l}\right)^{-1} B_{\nu}^{d}
$$

and its $H_{2}$ norm is

$$
\left\|T_{\nu}^{d z}\right\|_{2}^{2}=\frac{1}{2 \pi} \int_{-\pi}^{\pi} \operatorname{Tr}\left\{T_{\nu}^{d z}(\varsigma)^{*} T_{\nu}^{d z}(\varsigma)\right\} d \omega
$$

with $\varsigma=e^{j \omega}, \forall \nu \in \Gamma$. The following theorem gives a method to compute the state-feedback gain $K=\underset{K}{\operatorname{argmin}}\left\|T_{\nu}^{d z}\right\|_{2}^{2}$ which guarantees the asymptotical stability of the system (17) $\forall \nu \in \Gamma$.

Theorem 1: [4] If there exist symmetric positive definite matrices $W, P_{\nu}$, matrices $X, L$ and a scalar $\mu>0$ such that LMIs

$$
\begin{gathered}
\operatorname{Tr}(W)<\mu \\
{\left[\begin{array}{cc}
W & C^{z} X+D^{z u} L \\
(\star)^{\prime} & X+X^{\prime}-P_{\nu}
\end{array}\right] \succ 0} \\
{\left[\begin{array}{ccc}
P_{\nu} & A_{\nu} X+B_{\nu}^{u} L & B_{\nu}^{d} \\
(\star)^{\prime} & X+X^{\prime}-P_{\nu} & 0 \\
(\star)^{\prime} & (\star)^{\prime} & I
\end{array}\right] \succ 0}
\end{gathered}
$$

are verified $\forall \nu \in \Gamma$, then the controller (21) with $K=L X^{-1}$ guarantees the asymptotic stability of the system (17), with $\left\|T_{\nu}^{d z}\right\|_{2}^{2}<\mu$.

In order to choose the controller gain $K$ minimising the norm $\left\|T_{\nu}^{d z}\right\|_{2}^{2}$, we can solve the optimisation problem

$$
\left\{\begin{array}{l}
\min \mu \\
\text { under }(24)-(26)
\end{array} .\right.
$$

\section{RESULTS}

In this section, the control design described in the previous sections is applied to the ArcelorMittal HSM of Eisenhüttenstadt.
TABLE I

FAMILIES BOUNDS

\begin{tabular}{|c||c|c|c|c|}
\hline Family & $w$ & $h_{n}$ & $\sigma_{1}^{0}$ & $\sigma_{n}^{0}$ \\
\hline \hline 1 & $810-1200$ & $1.9-3$ & $22-65$ & $30-95$ \\
\hline 2 & $810-1200$ & $3-4.5$ & $22-65$ & $30-95$ \\
\hline 3 & $810-1200$ & $4.5-6.2$ & $22-65$ & $30-95$ \\
\hline 4 & $1200-1670$ & $1.9-3$ & $22-65$ & $30-95$ \\
\hline 5 & $1200-1670$ & $3-4.5$ & $22-65$ & $30-95$ \\
\hline 6 & $1200-1670$ & $4.5-6.2$ & $22-65$ & $30-95$ \\
\hline
\end{tabular}

TABLE II

COMPARISON OF THE COST FUNCTION VALUES

\begin{tabular}{|c|c|}
\hline Family & Cost function value \\
\hline \hline all & 752.8 \\
\hline 1 & 237.1 \\
\hline 2 & 66.3 \\
\hline 3 & 41.2 \\
\hline 4 & 251 \\
\hline 5 & 100.6 \\
\hline 6 & 48.9 \\
\hline
\end{tabular}

\section{A. Robust Steering Control Toolbox}

A Matlab toolbox (RSCT, [9]) has been developed for obtaining a systematic procedure to extend the steering control to different factories. It provides a user-friendly interface to compute the products database. Moreover, the algorithm described in section III is implemented. This allows to divide the database in $\mathcal{F}$ families and to compute the products corresponding to the vertices of each family $f \in \mathcal{F}$. Then, for each product, the corresponding reduced linear system is computed (see section II). These informations are necessary to design the controller gains $K^{f}$ using Theorem 1. The toolbox also includes the HSM simulator to analyse the system behaviour. The LMI problem (24)-(26) is solved using the free LMI solver SeDuMi [13] and the free Matlab toolbox YALMIP, which provides a simple interface for the most popular LMI solvers [8].

\section{B. Controllers Gain Computing}

After experimental trials, we decided to divide the whole database into $\mathcal{F}=6$ families, using the main parameters set $\mathcal{U}^{m}=\left\{w, h_{n}, \sigma_{1}^{0}, \sigma_{n}^{0}\right\}$. The families bounds are summarised in Table I.

The control system is designed in the discrete-time, with sample time $T_{s}=0.05 \mathrm{sec}$. Applying Theorem 1, we can find robust control gain $K^{f}$ for each family $f \in \Psi$.

The analysis of the cost function (27) shows that the described families choice improves the system performances. The results are summarised in Table II: in the first row the cost function value for the controller which stabilises the whole set of products is shown. The following rows show the cost function values corresponding to the controllers $K^{f}$ which stabilise only one family of products.

\section{Simulation Results}

Simulation results are shown for a product $p$ with $\mathcal{U}^{m}=\{967,2.02,27.9,40.1\}$. From Table I, $p$ belongs to 
the first family. Fig. 2 shows the output $Z$ evolution. The solid line represents the $Z$ evolution with the $\mathrm{H}_{2}$ control gain $K^{1}$. The dashed line shows the $Z$ evolution with a classic LQ control gain, designed for a medium product of family 1 . The dotted line shows the $Z$ evolution with a classic LQ control gain, designed for a medium product of the whole database. This last controller is presented in [3]. As expected, the

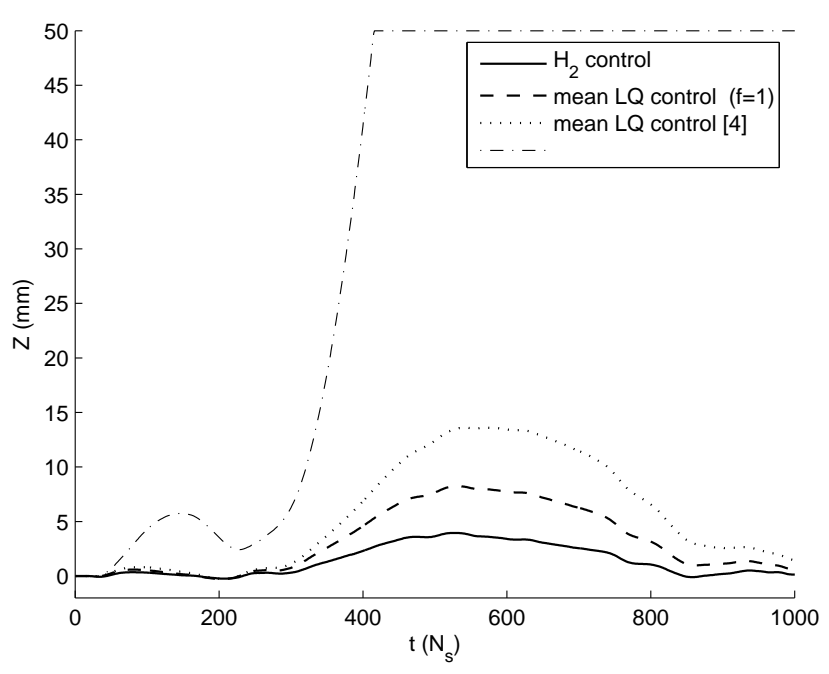

Fig. 2. Exit strip off-centre evolution

division of the whole database into more families improves the system performances. Moreover, the $\mathrm{H}_{2}$ robust controller takes into account the uncertain parameters and minimises the effects of the external perturbation, which is due to the coilbox vibrations. Then, results may still be improved. The last line, the dash-dotted one, shows the $Z$ evolution without control. Notice that in this case a saturation occurs: it means that the strip crashes against the HSM guides because of the elevated $Z$. The result is a decrease of the product quality and, in the worst case, the damage of the rolls.

\section{Industrial Implementation}

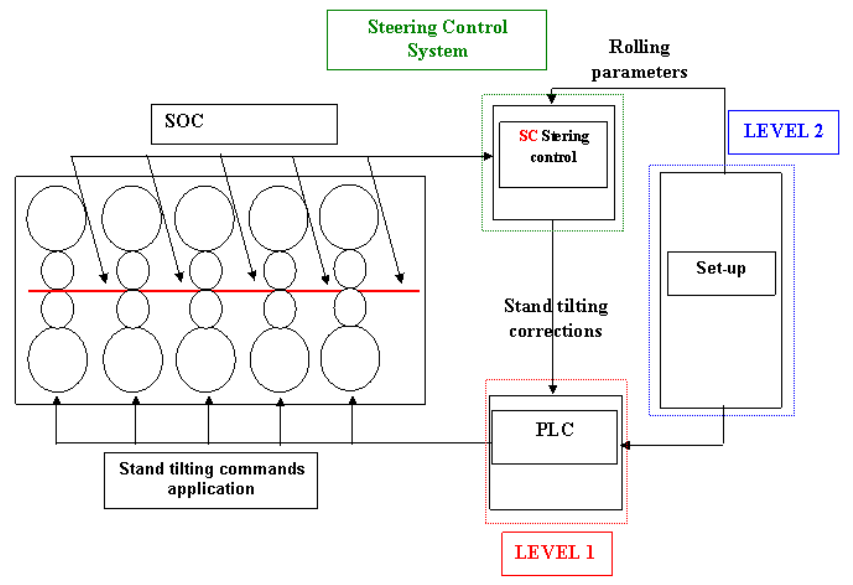

Fig. 3. Information global process at ArcelorMittal Eisenhüttenstadt HSM
The steering control system includes five cameras, the main computer SC and the data connection devices (Fig. 3). The cameras, which are DACOO4 delivered by Fife, are protected by a water-cooled housing (the strip can reach the $1000^{\circ} \mathrm{C}$ ) and mounted on dedicated vibration absorbers to avoid high accelerations. A Profibus connects each camera to the main computer, which is linked to the stand $P L C$ by Profibus as well. The main computer consists of a $3 \mathrm{GHz}$ Intel-P4 standard personal computer with integrated Profibus interface. The control system is developed in the $\mathrm{C}++$ language and works under the operating system Windows $X P$. A TCP/IP using an Ethernet connection realises the communication to the Level 2 system in order to load the rolling parameters. Filtering, active pixel selection and edge detection are done by FPGA devices, which are located directly on the cameras. This architecture allows to reduce the amount of data which is necessary transmitting to the main computer. An edge detection algorithm based on the gradient analyses is used in order to obtain a clear information concerning the strip off-centre values $Z$. During the operating phase of the control system, the stand tilting $u$ is obtained by multiplying the $Z$ values to the right controller gain $K^{f}$, computed off-line by $R S C T$. In order to avoid big values of $u$, due to measurement errors, a saturation function is applied before sending the stand tilting signal to the $P L C$. For the evaluation of the control system some indicators are computed and stored in a database.

\section{E. Experimental Results}

Some experimental result are presented here. In Fig. 4, the exit $Z$ evolution of two consecutive products with the same $\mathcal{U}^{m}=\{1510,2.02,59.1,72.5\}$ is shown. The solid line corresponds to the $Z$ evolution when the steering control is on whereas the dotted line corresponds to the open loop $Z$ evolution. Notice that the $Z$ value, which varies between -35 and $50 \mathrm{~cm}$ in open-loop, is maintained close to zero when the mill works in closed-loop. Then, a better quality of the rolled products is obtained. Moreover, the probability that the strip crashes against the guides decreases. Hence, the rolling process works in more safe conditions.

In Fig. 5, the exit strip off-centre evolution of 10 different products is shown. We chose the products in order to cover all the extreme combinations of the family 4 , with reference to the set of the main parameters $\mathcal{U}^{m}$. The initial coilbox perturbation, which can be deducted by the $Z$ initial values, varies between -25 and $30 \mathrm{~cm}$. However, the strip off-centre is kept between -15 and $20 \mathrm{~cm}$ during the whole rolling process. This fact proves the robustness of the designed control gain.

\section{CONCLUSION}

In this article, a $\mathrm{H}_{2}$ robust steering control is proposed in order to guarantee the stability of the HSM system and to improve its performances. Since a finishing mill treats products that can be very different, a significant database is created and divided into several families of products. A method to reduce the complexity of the problem exploiting the relation 


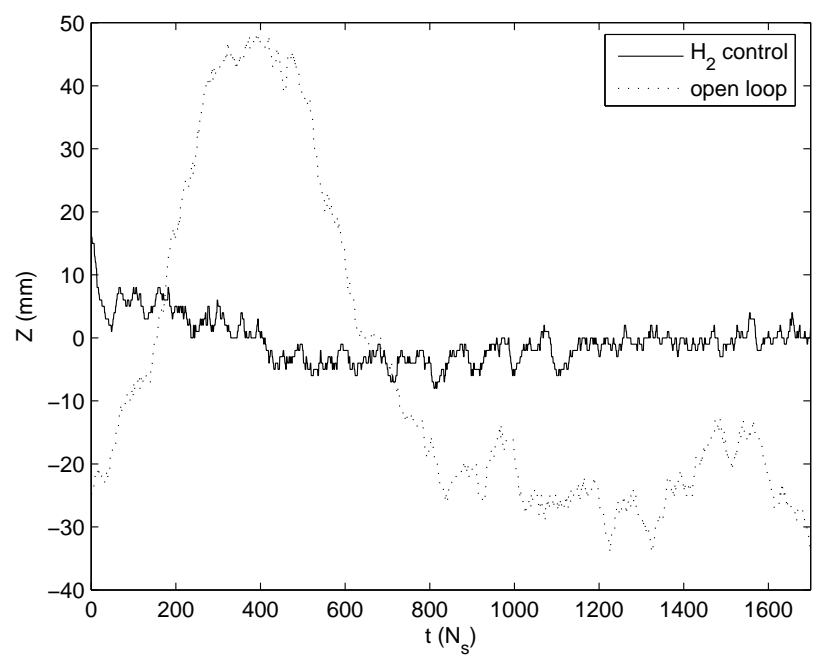

Fig. 4. Exit strip off-centre evolution

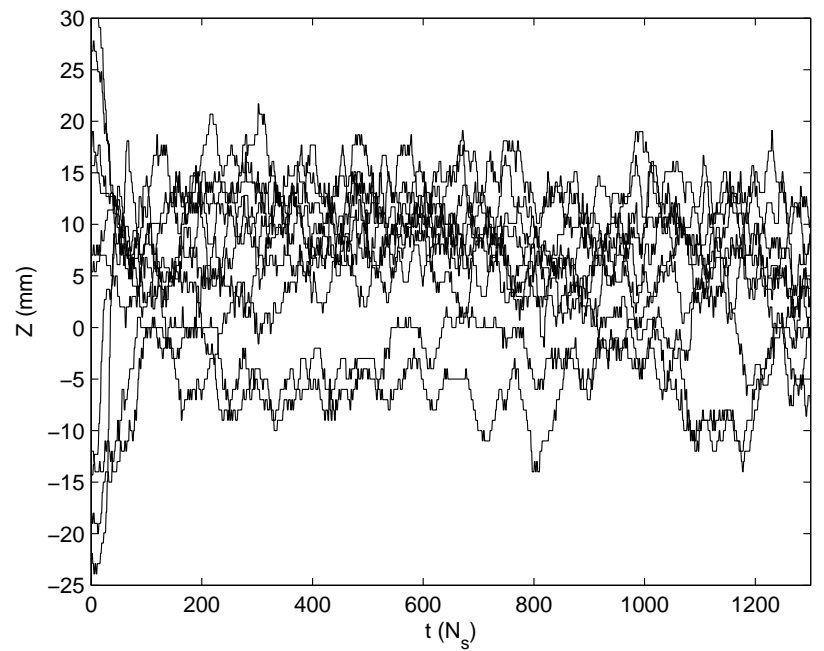

Fig. 5. Exit strip off-centre evolution (family 4)

between the different products parameters is also presented. This method yields to a convex stabilisation problem. Then, for each family, a different LMI-based controller is designed.

We proposed a division of the whole set of products into 6 families and we showed that the corresponding designed controllers guarantee the asymptotic stability of the closedloop system. Moreover, the presented control law reduces the strip off-centre improving the product quality and the system safety. Also if the results that we presented concern the ArcelorMittal HSM of Eisenhüttenstadt, the described steering control design is adaptable to any plant.

The next step consists in solving the tail end phase stabilisation problem. The tail end phase is the last phase of the hot rolling process: the strip leaves the stands one after the other. Each time the strip leaves a stand the system dynamics changes. Hence, the HSM system can be described as a switched system [7].

\section{REFERENCES}

[1] C.B. Barber, D.P. Dobkin, and H.T. Huhdanpaa. The quickhull algorithm for convex hulls. ACM Transactions on Mathematical Software, 22(4):469-483, 1996.

[2] S. Boyd, L.E. Ghaoui, E. Feron, and V. Balakrishnan. Linear Matrix Inequalities in system and control theory. Society for Industrial and Applied Mathematics, 1994.

[3] J. Daafouz, R. Bonidal, C. Iung, P. Szczepanski, N. Naumann, and U. Koschack. New steering control at EKO Stahl finishing mill. In The Iron \& Steel Technology Conference and Exposition, 2008.

[4] M.C. de Oliveira, J.C. Geromel, and J. Bernussou. An LMI optimization approach to multiobjective and robust $\mathrm{H}_{\infty}$ controller design for discrete-time systems. International Journal of Control, 75:666-679, 2002.

[5] Y. Furukawa, S. Fujii, and H. Taoka. Application of steering control in hot strip mill. Tetsu-to-Hagane (Journal of the Iron and Steel Institute of Japan), 78(8):141-144, 1992.

[6] P. Kokotovic, H.K. Khalil, and J. O'Reilly. Singular perturbation methods in control: analysis and design. Academic Press, 1986.

[7] D. Liberzon. Switching in systems and control. Birkhäuser, 2003.

[8] J. Löefberg. YALMIP : A toolbox for modeling and optimization in MATLAB. In IEEE international symposium on computer aided control systems design, 2004.

[9] I. Malloci, R. Bonidal, P. Szczepanski, J. Daafouz, and C. Iung. Robust Steering Control Toolbox. Internal Rapport ArcelorMittal, 2008.

[10] Y. Marushita, H. Ikeda, K. Yano, and S. Shindo. Advanced control method of steering on the hot rolling mill. In 5th IFAC Automation in Mining, Mineral, and Metal Processing, 2001.

[11] Y. Okamura and I. Hoshino. State feedback control of the strip steering for aluminum hot rolling mill. Control Engineering Practice, 5(8):1035-1042, 1997.

[12] M.J. Steeper and G.D. Park. Development of steering control system for reversing hot mills. Iron and steel engineer, 75(11):21-24, 1998.

[13] J.F. Sturm. Using SeDuMi 1.02, a Matlab Toolbox for optimisation over symmetric cones. Optimization Methods and Software, 1112:625-653, 1999. 